\title{
The Lamé equation in parametric resonance after inflation
}

\author{
F. Finkel ${ }^{1, *}$ A. González-López, ${ }^{1, \dagger}$ A. L. Maroto, ${ }^{2, \sharp}$ and M. A. Rodríguez ${ }^{1, \S}$ \\ ${ }^{1}$ Departmento de Física Teórica II, Facultad de Ciencias Físicas, Universidad Complutense, 28040 Madrid, Spain \\ ${ }^{2}$ Departamento de Física Teórica I, Facultad de Ciencias Físicas, Universidad Complutense, 28040 Madrid, Spain
}

(Received 16 June 2000; revised manuscript received 13 July 2000; published 17 October 2000)

\begin{abstract}
We show that the most general inflaton potential in Minkowski spacetime for which the differential equation for the Fourier modes of the matter fields reduces to Lamé's equation is of the form $V(\phi)=\lambda \phi^{4} / 4+K \phi^{2} / 2$ $+\mu /\left(2 \phi^{2}\right)+V_{0}$. As an application, we study the preheating phase after inflation for the above potential with $K=0$ and arbitrary $\lambda, \mu>0$. For certain values of the coupling constant between the inflaton and the matter fields, we calculate the instability intervals and the characteristic exponents in closed form.
\end{abstract}

PACS number(s): 98.80.Cq, 98.80.Hw

\section{INTRODUCTION}

The phenomenon of parametric resonance plays a fundamental role in modern theories of preheating after inflation [1-3]. At the end of the slow-roll phase, the inflaton field starts oscillating around the minimum of the potential in a coherent way. If this field is coupled to some other matter fields, the periodic evolution can give rise to an explosive production of matter quanta thanks to the resonant amplification of vacuum fluctuations. This production is typically characterized by the exponential growing of the occupation number of those states whose momentum lies within certain resonance bands. This effect is essentially nonperturbative, and the corresponding production of particles is much more efficient than the traditional perturbative decay of the inflaton field during reheating. However, this nonperturbative character makes it extremely difficult to obtain exact results in most inflation models and, as a consequence, one must rely on numerical computations in order to obtain information about spectra or time evolution of the particle production. Despite this fact, a few models are known for which it is possible to obtain the width of the resonance bands and the characteristic exponents in an analytical fashion. In particular, the pure quadratic potential $V(\phi)=m^{2} \phi^{2} / 2$ for the inflaton field in Minkowski space yields the Mathieu equation for the Fourier modes of a scalar field $\chi$ coupled to the inflaton as $g^{2} \phi^{2} \chi^{2} / 2[4,1]$. In an expanding background, the quartic inflaton potential $V(\phi)=\lambda \phi^{4} / 4$ leads to the Lamé equation for the corresponding matter fields modes [5]. This equation also appears in connection with some other combinations of these inflaton potentials, such as $V(\phi)=\left(\phi^{2}-\sigma^{2}\right)^{2}$ [6]. Parametric resonance driven by the latter potential has also been proposed as a mechanism for the generation of longwavelength pion modes from disoriented chiral condensates (DCC) [7].

The relevance of the Lamé equation in the study of parametric resonance stems from its unique analytical properties, which makes it possible to compute the resonance bands and

\footnotetext{
*Electronic address: federico@ciruelo.fis.ucm.es

†Electronic address: artemio@eucmos.sim.ucm.es

*Electronic address: maroto@eucmax.sim.ucm.es

${ }^{\S}$ Electronic address: rodrigue@eucmos.sim.ucm.es
}

the associated characteristic exponents in closed form $[5,6]$. In this paper we extend the above results and derive the most general inflaton potential in Minkowski spacetime for which the corresponding matter modes equation reduces to the Lamé form. The resulting potential possesses a term of the form $\phi^{-2}$, appearing typically in the context of certain supersymmetric inflation models, see Refs. [8,9].

The paper is organized as follows. In Sec. II we present our main result, obtaining the most general potential for the inflaton field in a Minkowskian background leading to the Lamé equation for the matter fields via the usual $g^{2} \phi^{2} \chi^{2} / 2$ coupling. For a certain choice of the parameters of this potential, we explicitly determine the scaling factors necessary to reduce the equation for the Fourier modes of the matter fields to the Lamé equation. In Sec. III, we determine the range of values of these parameters compatible with the slow-roll approximation and the amplitude of the density perturbations observed by the Cosmic Background Explorer (COBE). For several values of the coupling constant $g$ we compute (using the results of Refs. [5,6]) the resonance bands, the corresponding characteristic exponents, and the number density of particles produced. Our results show that the particle production in the preheating phase remains unchanged if a term $\mu /\left(2 \phi^{2}\right)$ is added to the $\lambda \phi^{4} / 4$ model for a broad range of values of the parameter $\mu$. We finally summarize in Sec. IV the main results of our paper.

\section{THE INFLATON POTENTIAL}

Let us consider the following Lagrangian density for the inflaton field $\phi$ coupled to a scalar massless matter field $\chi$ :

$$
\mathcal{L}=\frac{1}{2} g^{\mu \nu} \partial_{\mu} \phi \partial_{\nu} \phi+\frac{1}{2} g^{\mu \nu} \partial_{\mu} \chi \partial_{\nu} \chi-V(\phi)-\frac{g^{2}}{2} \phi^{2} \chi^{2}
$$

We will assume our background fields to be homogeneous and isotropic, i.e., the space-time metric is of the FriedmannRobertson-Walker form $d s^{2}=d t^{2}-a^{2}(t) d \mathbf{x}^{2}$, and the inflaton field $\phi(t)$ depends only on time. The corresponding classical equations of motion for the different fields are

$$
\ddot{\phi}+3 H \dot{\phi}+V^{\prime}(\phi)=0
$$




$$
\ddot{\chi}+3 H \dot{\chi}-\frac{1}{a^{2}} \partial_{i} \partial_{i} \chi+g^{2} \phi^{2} \chi=0,
$$

where $H=\dot{a} / a$, and the prime denotes differentiation with respect to the argument of $V$. Note that we are neglecting back reaction effects from the $\chi$ fields in the inflaton equation (2) assuming that the expectation value $\left\langle\chi^{2}\right\rangle$ is negligible during the first inflaton oscillations.

Let us rescale the field as $\hat{\phi}=a \phi, \hat{\chi}=a \chi$, and work in conformal $\eta$ time defined by $a d \eta=d t$. It is then possible to rewrite the equations of motion as

$$
\begin{gathered}
\frac{d^{2} \hat{\phi}}{d \eta^{2}}+a^{3} V^{\prime}\left(a^{-1} \hat{\phi}\right)-\frac{1}{a} \frac{d^{2} a}{d \eta^{2}} \hat{\phi}=0, \\
\frac{d^{2} \hat{\chi}}{d \eta^{2}}-\partial_{i} \partial_{i} \hat{\chi}+g^{2} \hat{\phi}^{2} \hat{\chi}-\frac{1}{a} \frac{d^{2} a}{d \eta^{2}} \hat{\chi}=0 .
\end{gathered}
$$

In the particular case in which the potential is $V(\phi)$ $=\lambda \phi^{4} / 4$, it can be shown [5] that the scale factor grows as $a(\eta) \propto \eta$, and hence the last terms in Eqs. (4) and (5) vanish, while the term $a^{3} V^{\prime}\left(a^{-1} \hat{\phi}\right)$ reduces to $\lambda \hat{\phi}^{3}$. The equations of motion are thus expressed in Minkowskian form in terms of the rescaled fields. However, this is not true for an arbitrary potential, and for that reason in the following we will concentrate only in the Minkowskian limit $(a=1)$. This can be considered as a first approximation to the full problem and, in particular, it will allow us to obtain analytic results in some cases. Nevertheless, we expect that our results will carry over to an expanding universe with minimal quantitative changes (though rather important analytic differences) when the potential differs from $\lambda \phi^{4} / 4$ by a small perturbation.

Let us then consider the equations of motion in Minkowski space-time

$$
\begin{array}{r}
\frac{d^{2} \phi}{d t^{2}}+V^{\prime}(\phi)=0, \\
\frac{d^{2} \chi}{d t^{2}}-\partial_{i} \partial_{i} \chi+g^{2} \phi^{2} \chi=0 .
\end{array}
$$

We shall now derive the most general inflaton potential $V(\phi)$ for which the differential equation for the Fourier modes of the matter fields

$$
\frac{d^{2} \chi_{k}}{d t^{2}}+\left(k^{2}+g^{2} \phi^{2}\right) \chi_{k}=0
$$

reduces to the Lamé equation

$$
\frac{d^{2} X_{k}}{d x^{2}}+\left(\mathcal{E}-m n(n+1) \operatorname{sn}^{2} x\right) X_{k}=0
$$

under a change of scale

$$
t=\tau x, \quad \phi(t)=\phi_{0} f(x), \quad \chi_{k}(t)=X_{k}(x) .
$$

In the latter equations $m=\kappa^{2} \in(0,1)$ is the square of the modulus of the elliptic sine function $\operatorname{sn} x \equiv \operatorname{sn}(x, \kappa)$. Comparing Eqs. (8) and (9) we see that we can take without loss of generality

$$
f(x)= \pm\left(\beta-\operatorname{sn}^{2} x\right)^{1 / 2}
$$

and

$$
\mathcal{E}=\tau^{2}\left(k^{2}+\beta g^{2} \phi_{0}^{2}\right), \quad m n(n+1)=\tau^{2} g^{2} \phi_{0}^{2} .
$$

Note that $\beta \geqslant 1$ for $f$ to be real for all $x \in \mathbf{R}$.

It follows from Eq. (6) that

$$
\frac{1}{2}\left(\frac{d \phi}{d t}\right)^{2}+V(\phi)=E,
$$

where the integration constant $E$ is the energy density of the inflaton field. Using the well-known identities

$$
\frac{d}{d x} \operatorname{sn} x=\operatorname{cn} x \operatorname{dn} x
$$

and

$$
\operatorname{cn}^{2} x=1-\beta+f^{2}(x), \quad \operatorname{dn}^{2} x=1-m \beta+m f^{2}(x),
$$

we immediately show that

$$
\frac{1}{2}\left(\frac{d f}{d x}\right)^{2}+U(f)=E_{f},
$$

with $U$ and $E_{f}$ given by

$$
\begin{aligned}
U(f) & =\frac{m f^{4}}{2}+\frac{1}{2}(1+m-3 m \beta) f^{2}+\frac{\beta(\beta-1)(1-m \beta)}{2 f^{2}}, \\
E_{f} & =-\frac{1}{2}\left[3 m \beta^{2}-2(1+m) \beta+1\right] .
\end{aligned}
$$

Comparison of Eqs. (13) and (14) using Eq. (10) shows that the inflaton potential $V(\phi)$ must be of the form [16]

$$
V(\phi)=\lambda \frac{\phi^{4}}{4}+K \frac{\phi^{2}}{2}+\frac{\mu}{2 \phi^{2}}+V_{0},
$$

where the potential coefficients $(\lambda, K, \mu)$ and the energy density $E$ are related to the parameters $\left(\beta, m, \tau, \phi_{0}\right)$ through the following equations:

$$
\begin{aligned}
& \lambda=\frac{2 m}{\tau^{2} \phi_{0}^{2}}, \quad K=\frac{1}{\tau^{2}}(1+m-3 m \beta), \\
& \mu=\frac{\phi_{0}^{4}}{\tau^{2}} \beta(\beta-1)(1-m \beta), \\
& E=-\frac{\phi_{0}^{2}}{2 \tau^{2}}\left[3 m \beta^{2}-2(1+m) \beta+1\right]+V_{0} .
\end{aligned}
$$


Note that the positivity of $\lambda$ forces $\mu$ to be non-negative for $V$ to have a global minimum. We have thus shown that, in order for Eq. (8) to reduce under scaling to Lamé's equation (9), the inflaton potential $V(\phi)$ must necessarily be of the form (15).

One must now prove that any potential of the form (15) leads to Lamé's equation (9). For this to be the case, Eqs. (16) -(18) should have a solution for arbitrary values of $\lambda>0, K, \mu \geqslant 0$ and $E>V_{\min }$, where $V_{\min }$ is the minimum value of the potential (15) (the value $E=V_{\text {min }}$ must be discarded, since then $\phi$ reduces to the trivial constant solution $\left.\phi=\phi_{\min }\right)$. We shall verify this only for the case $K=0$, which we shall study in detail in the following sections.

For $K=0$ the second Eq. (16) can be used to solve for $\beta$ in terms of $m$, obtaining

$$
\beta=\frac{1}{3}\left(1+\frac{1}{m}\right)
$$

The condition $\beta \geqslant 1$ then implies that

$$
0<m \leqslant \frac{1}{2} \text {. }
$$

When $0<m<1 / 2$ the remaining equations (17), (18) are easily solved, with the following result:

$$
\begin{aligned}
\tau^{2} & =\frac{2}{3}\left(\lambda^{2} \mu\right)^{-1 / 3} D(m), \quad \phi_{0}^{2}=\left(\frac{\mu}{\lambda}\right)^{1 / 3} \frac{3 m}{D(m)}, \\
E & =V_{1} \frac{m^{2}-m+1}{D^{2}(m)}+V_{0},
\end{aligned}
$$

where

$$
D(m)=\left[(1+m)(2-m)\left(\frac{1}{2}-m\right)\right]^{1 / 3}
$$

and $V_{1}=3 / 4\left(\lambda \mu^{2}\right)^{1 / 3}$ is the minimum of the potential (15) for $K=V_{0}=0$. Since

$$
\widetilde{E}(m)=\frac{m^{2}-m+1}{D^{2}(m)}
$$

grows monotonically in the interval $m \in(0,1 / 2)$, with $\widetilde{E}(0)$ $=1$ and $\lim _{m \rightarrow 1 / 2} \widetilde{E}(m)=+\infty$, it follows that Eq. (21) uniquely determines $m \in(0,1 / 2)$ for arbitrary values of $E$ $>V_{1}+V_{0}=V_{\min }$. Equation (20) then yields $\tau$ and $\phi_{0}$ for arbitrary $\lambda, \mu>0$. For $m=1 / 2$ Eqs. (16)-(18) simplify to

$$
\lambda=\frac{1}{\tau^{2} \phi_{0}^{2}}, \quad \mu=0, \quad E=\frac{\phi_{0}^{2}}{4 \tau^{2}} .
$$

These are the equations obtained in Ref. [5] for the $\lambda \phi^{4} / 4$ potential, which again uniquely determine $\tau$ and $\phi_{0}$ in terms of $\lambda>0$ and $E>V_{\min }=0$. In fact, for $m=1 / 2 \mathrm{Eq}$. (19) implies that $\beta=1$, so that Eq. (11) yields $f^{2}(x)=\operatorname{cn}^{2}(x, 1 / \sqrt{2})$.

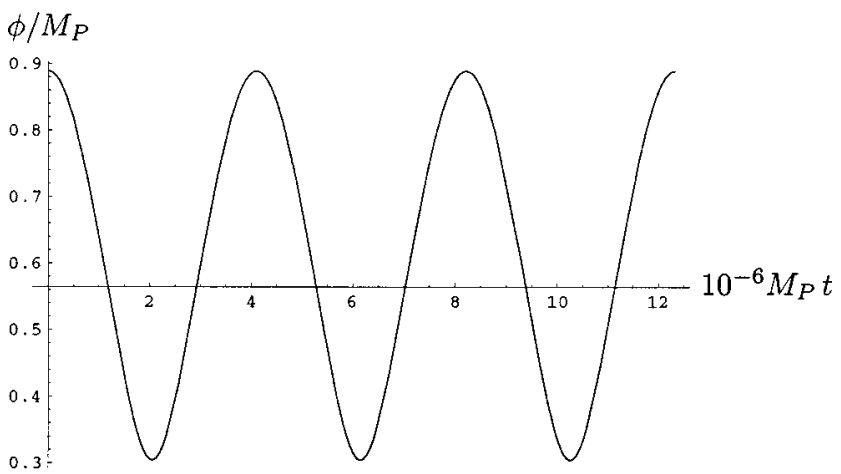

FIG. 1. Oscillations after inflation of the inflaton field $\phi$ (in units of $M_{P}$ ) as a function of time $t$ (in units of $10^{6} / M_{p}$ ) computed from the exact formulas (10), (11). We have taken $\lambda=9.091$ $\times 10^{-13}, \mu^{1 / 6}=5.553 \times 10^{-3} M_{P}, m=0.417$, corresponding to $\widetilde{\alpha}$ $=1$; see the discussion in the text for details. The horizontal axis is placed at $\phi_{\min } / M_{p}=1 / \sqrt{\pi}$.

\section{EXACT RESULTS FOR THE $\lambda \phi^{4} / 4+\mu /\left(2 \phi^{2}\right)$ MODEL}

Potential terms of the form $\mu \phi^{-p}$ have been considered in different contexts in the literature. They appear in hybrid inflation models [10], or in the so-called intermediate inflation [11]. But it is probably in the context of supersymmetric models of inflation where this kind of terms is more relevant. In fact, these contributions arise generically due to nonperturbative effects in supersymmetric gauge theories [12], the scale $\mu$ and the exponent $p$ depending on the particular gauge group in the theory. Inflationary models based on this kind of potentials have been studied in Refs. [8,9].

Motivated by the connection with the Lamé equation established in the previous section, we shall consider an effective potential during inflation and preheating of the form

$$
V(\phi)=V_{0}+\lambda \frac{\phi^{4}}{4}+\frac{\mu}{2 \phi^{2}}
$$

We shall derive an analytic expression for the instability intervals and the characteristic exponents for certain values of the ratio $g^{2} / \lambda$. In principle, higher-order terms could appear in the full potential, but we will assume that their effect is negligible during inflation and preheating. We could also have considered the contribution of a mass term as shown in the previous section, but in order to obtain closed expressions we will ignore it in what follows.

The minimum of the potential is placed at $\phi_{\min }=(\mu / \lambda)^{1 / 6}$ $=\alpha$, while the constant $V_{0}$ must be taken as $V_{0}=-V_{1}$, i.e. $V_{\min }=0$, for the cosmological constant to vanish. The inflaton field $\phi$ oscillates around $\phi_{\min }$ with amplitude $\alpha(\sqrt{1+m}$ $-\sqrt{1-2 m}) /(2 \sqrt{D(m)})$ and period $2 \tau K(\kappa)$, where $K(\kappa)$ is the complete elliptic integral of the first kind [13]. Note that, in contrast to the pure $\lambda \phi^{4} / 4$ model, $\phi_{\min }$ is nonzero and the oscillations are not symmetric about $\phi_{\min }$ (see Fig. 1).

Let us introduce the following notation: $\widetilde{\phi}=\phi / \alpha$ and $\widetilde{\alpha}$ $=\sqrt{\pi} \alpha / M_{P}$. The slow-roll parameters $\epsilon$ and $\eta$ for this model are given by 


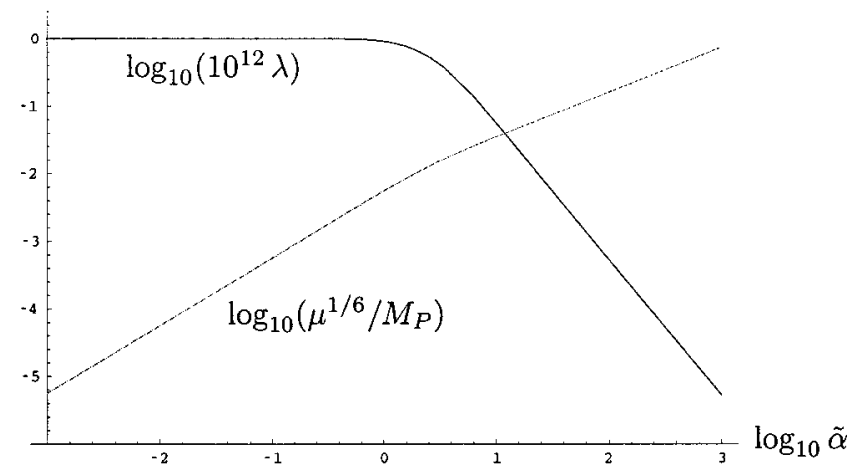

FIG. 2. Log-log plots of $10^{12} \lambda$ and $\mu^{1 / 6} / M_{P}$ versus $\widetilde{\alpha}$ for $10^{-3} \leqslant \widetilde{\alpha} \leqslant 10^{3}$.

$$
\epsilon=\frac{M_{P}^{2}}{16 \pi}\left(\frac{V^{\prime}}{V}\right)^{2}=\frac{1}{\widetilde{\alpha}^{2}}\left(\frac{\widetilde{\phi}^{4}+\widetilde{\phi}^{2}+1}{\widetilde{\phi}^{5}+\widetilde{\phi}^{3}-2 \widetilde{\phi}}\right)^{2}
$$

and

$$
\eta=\frac{M_{P}^{2}}{8 \pi} \frac{V^{\prime \prime}}{V}=\frac{3}{2 \widetilde{\alpha}^{2}} \frac{\widetilde{\phi}^{6}+1}{\widetilde{\phi}^{8}-3 \widetilde{\phi}^{4}+2 \widetilde{\phi}^{2}} .
$$

The end of inflation occurs when the slow-roll approximation breaks down, i.e., for $\epsilon \simeq 1$ or $\eta \simeq 1$. In the case $\widetilde{\alpha} \ll 1$ the value $\widetilde{\phi}_{\text {end }}$ at the end of inflation is given to a very good approximation by $\widetilde{\phi}_{\text {end }} \simeq \sqrt{3 / 2} \widetilde{\alpha}^{-1}$. However for $\widetilde{\alpha} \gg 1$ the corresponding value behaves as $\widetilde{\phi}_{\text {end }} \simeq 1+1 / 2 \widetilde{\alpha}^{-1}$. The initial value $\widetilde{\phi}_{60}$ corresponding to $60 e$-folds before inflation ends is determined by the condition

$$
60 \simeq-\frac{8 \pi}{M_{P}^{2}} \int_{\phi_{60}}^{\phi_{\text {end }}} \frac{V}{V^{\prime}} d \phi .
$$

It follows that $\widetilde{\phi}_{60} \simeq \sqrt{123 / 2} \widetilde{\alpha}^{-1}$ if $\widetilde{\alpha} \ll 1$ and $\widetilde{\phi}_{60} \simeq 1$ $+11 / 2 \widetilde{\alpha}^{-1}$ if $\widetilde{\alpha} \gg 1$. Accordingly, the amplitude of the density perturbations at $\phi=\phi_{60}$ can be written as

$$
\begin{aligned}
\delta_{H}(k) & =\left.\frac{16}{5} \sqrt{\frac{2 \pi}{3}} \frac{V^{3 / 2}}{M_{P}^{3}\left|V^{\prime}\right|}\right|_{\phi=\phi_{60}} \\
& =\frac{2}{5 \pi} \sqrt{\frac{2 \lambda}{3}} \widetilde{\alpha}^{3} \frac{\left(\widetilde{\phi}_{60}^{6}-3 \widetilde{\phi}_{60}^{2}+2\right)^{3 / 2}}{\widetilde{\phi}_{60}^{6}-1} .
\end{aligned}
$$

We thus get $\delta_{H}(k) \simeq(\sqrt{41 \lambda} / \pi)\left(123 / 5+96 / 41 \widetilde{\alpha}^{4}\right)$ for $\widetilde{\alpha} \ll 1$ and $\delta_{H}(k) \simeq 242 /(5 \pi) \sqrt{2 \lambda} \widetilde{\alpha}$ for $\widetilde{\alpha} \gg 1$. The COBE normalization $\delta_{H}(k) \simeq 5 \times 10^{-5}$ determines $\lambda$ (and thus $\mu$ ) as a function of $\widetilde{\alpha}$, see Fig. 2. In the limit $\widetilde{\alpha}=0$ we get $\lambda$ $\simeq 10^{-12}$, while $\lambda \simeq 5 \times 10^{-12} \widetilde{\alpha}^{-2}$ for $\widetilde{\alpha} \gg 1$. Since $\mu^{1 / 6} \lesssim M_{P}$ we get $\lambda \gtrsim 2 \times 10^{-18}$ when $\widetilde{\alpha} \gg 1$. This in turn implies that $\widetilde{\alpha} \lesssim 2 \times 10^{3}$.

The value of $m$ is obtained from Eq. (21), with the energy density of the inflaton field given by

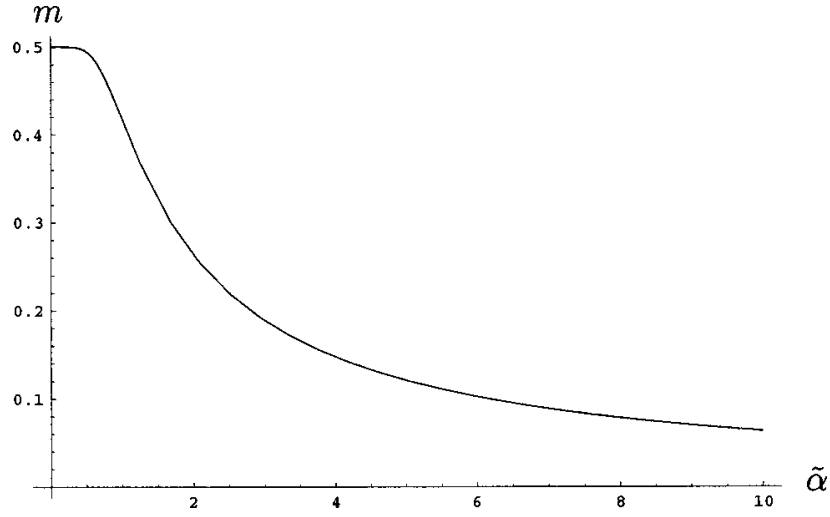

FIG. 3. Value of the square modulus $m$ as a function of $\widetilde{\alpha}$.

$$
E=V\left(\phi_{\text {end }}\right)=V_{1} \frac{\widetilde{\phi}_{\mathrm{end}}^{6}-3 \widetilde{\phi}_{\mathrm{end}}^{2}+2}{3 \widetilde{\phi}_{\mathrm{end}}^{2}} .
$$

For $\widetilde{\alpha} \ll 1$, to a very good approximation we have $\widetilde{E}$ $\simeq 3 / 4 \widetilde{\alpha}^{-4}$. From Eq. (22) we thus get $m \simeq 1 / 2-4 / 9 \widetilde{\alpha}^{6}$. On the other hand, for $\widetilde{\alpha} \gg 1$ we have $\widetilde{E} \simeq 1+\widetilde{\alpha}^{-2}$, leading to $m \simeq 2 / 3 \widetilde{\alpha}^{-1}$. In Fig. 3 we present the plot of $m$ as a function of $\widetilde{\alpha}$ for $\widetilde{\alpha}$ between 0 and 10 .

If $n$ is a positive integer, the Lamé equation (9) possesses exactly $n+1$ instability zones as the parameter $\mathcal{E}$ takes values on the real line, whose corresponding solutions grow exponentially at either $\pm \infty$ [14]. However, only those instability bands for which the squared momentum $k^{2}$ given by Eq. (12) is non-negative are physically significant. According to Floquet's theorem, the solutions of the Lamé equation in an instability band can be written as $X_{k}(x)$ $=e^{ \pm \mu_{k} x} P_{k}(x)$, where $P_{k}(x)$ is a periodic function and the characteristic exponent $\mu_{k}$ has a nonzero real part. The occupation number for particles with momentum $k$ produced in the preheating phase can be estimated in terms of the characteristic exponent as $N_{k}(t) \sim e^{2 \mu_{k} t / \tau}$ [6]. Remarkably, for integer $n$ the characteristic exponent for the Lamé equation is given by an exact formula involving a quadrature which, at least for the lowest values of $n$, can be expressed in terms of elliptic integrals $[5,6]$. We shall omit here most details and quote from the above references only the main steps for the derivation of this formula.

The key ingredient for obtaining the formula is the construction of an exact expression for the product of two linearly independent solutions of the Lamé equation [17]. The product of two such solutions satisfies the third-order differential equation given by

$$
\begin{aligned}
2 p(z) & M^{\prime \prime \prime}(z)+3 p^{\prime}(z) M^{\prime \prime}(z)+\left[p^{\prime \prime}(z)\right. \\
& +2(\mathcal{E}+m n(n+1)(z-1))] M^{\prime}(z) \\
& +m n(n+1) M(z)=0,
\end{aligned}
$$

where $z=\operatorname{cn}^{2} x$, and

$$
p(z)=(1-m) z+(2 m-1) z^{2}-m z^{3} .
$$


If $n$ is a nonnegative integer, Eq. (29) is satisfied by a suitable polynomial of degree $n$, which (following [6]) shall be written as

$$
M_{(n)}(z)=\sum_{i=0}^{n} a_{i}^{(n)} z^{n-i}
$$

with the normalization condition $a_{0}^{(n)}=1$. Since $z$ is a periodic function of $x$, the polynomial $M_{(n)}(z)$ coincides with the product of two linearly independent solutions of the Lamé equation (9) when $\mathcal{E}$ belongs to an instability zone. It was shown in Refs. $[5,6]$ that the characteristic exponent is determined by the definite integral

$$
\mu_{k}=\frac{C_{(n)}}{2 K(\kappa)} \int_{0}^{1} \frac{d z}{\sqrt{p(z)} M_{(n)}(z)},
$$

where

$$
C_{(n)}^{2}=(m n(n+1)-\mathcal{E})\left(a_{n}^{(n)}\right)^{2}+(m-1) a_{n-1}^{(n)} a_{n}^{(n)} .
$$

The instability intervals can be obtained imposing that the right-hand side of Eq. (32) be positive. The sign of $C_{(n)}$ is chosen so that the real part of $\mu_{k}$ is positive if $\mathcal{E}$ lies in an instability zone.

If all the roots of the polynomial $M_{(n)}(z)$ are real and different, the definite integral in Eq. (31) can be expressed in terms of elliptic integrals. Indeed, let $z=1-y$, and let the constants $\beta_{i}, D_{i}, i=1, \ldots, n$, be defined by

$$
\frac{1}{M_{(n)}(1-y)}=\sum_{i=1}^{n} \frac{D_{i}}{1-\beta_{i}^{-1} y} .
$$

The formula (31) for the characteristic exponent $\mu_{k}$ then reduces to

$$
\mu_{k}=\frac{C_{(n)}}{K(\kappa)} \sum_{i=1}^{n} D_{i} \hat{\Pi}\left(\beta_{i}^{-1} \mid \kappa\right)
$$

with

$$
\hat{\Pi}\left(\beta_{i}^{-1} \mid \kappa\right)= \begin{cases}\Pi\left(\beta_{i}^{-1} \mid \kappa\right) & \text { if } \beta_{i}^{-1}<1, \\ K(\kappa)-\Pi\left(\beta_{i} \kappa^{2} \mid \kappa\right) & \text { if } \beta_{i}^{-1}>1,\end{cases}
$$

where $\Pi(s \mid \kappa)$ is the complete elliptic integral of the third kind [13].

We shall now compute the characteristic exponent for $n$ $=1,2,3$ using Eq. (34). It shall be convenient to define the dimensionless momentum $\widetilde{k}=k / M_{P}$. Using Eq. (20) and the definition of $\widetilde{\alpha}$, Eq. (12) becomes

$$
\mathcal{E}=\frac{1}{3}\left(\frac{2 \pi D(m)}{\lambda \widetilde{\alpha}^{2}} \widetilde{k}^{2}+(m+1) n(n+1)\right) .
$$

For $n=1$, the Lamé equation (9) possesses two instability zones, namely $\mathcal{E} \in(-\infty, m)$ and $\mathcal{E} \in(1,1+m)$; see for instance Ref. [15]. The first instability zone is not admissible,

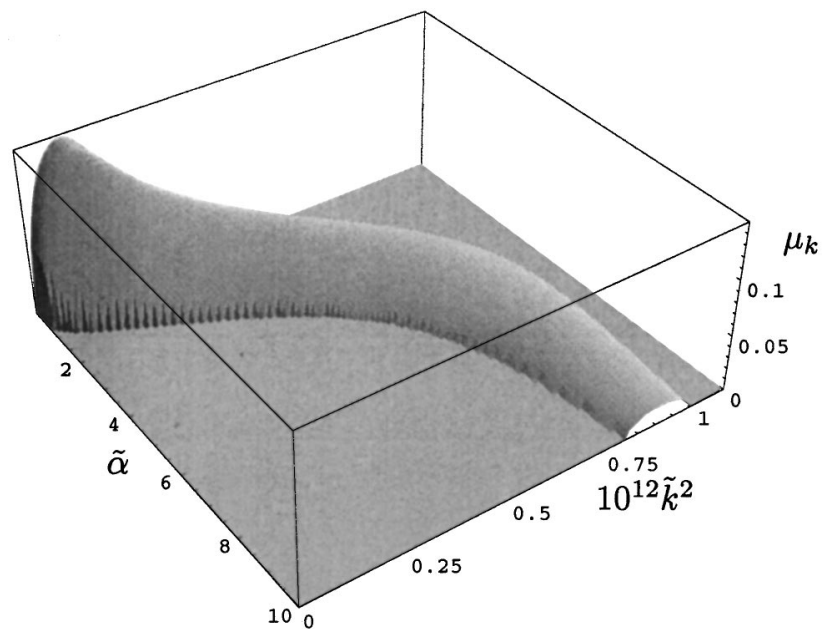

FIG. 4. Characteristic exponent as a function of $\widetilde{\alpha}$ and $10^{12} \widetilde{k}^{2}$ for $n=1$.

since it would force the momentum $k$ to take imaginary values. Using Eq. (36), the second instability zone leads to the resonance condition

$$
\frac{\lambda \widetilde{\alpha}^{2}(1-2 m)}{2 \pi D(m)}<\widetilde{k}^{2}<\frac{\lambda \widetilde{\alpha}^{2}(m+1)}{2 \pi D(m)} .
$$

Thus for $m \neq 1 / 2$ (i.e., for $\widetilde{\alpha} \neq 0$ ), there is an initial threshold for the resonant values of the momentum. If the $\chi$ field had a mass $m_{\chi}$, the instability interval (37) would be shifted by an amount $-m_{\chi}^{2} / M_{P}^{2}$. For $m_{\chi}$ large enough, this could result in the disappearance of the threshold, or even of the whole interval.

The polynomial $M_{(1)}(z)$ is given by

$$
M_{(1)}(z)=z+\frac{1-\mathcal{E}}{m}
$$

Therefore

$$
\beta_{1}^{-1}=D_{1}=\frac{m}{1+m-\mathcal{E}}
$$

and

$$
C_{(1)}^{2}=\frac{1}{m^{2}}(\mathcal{E}-m)(\mathcal{E}-1)(1+m-\mathcal{E}) .
$$

Note that $\beta_{1}^{-1}>1$ in the second instability zone. In Fig. 4 we plot the characteristic exponent as a function of $\widetilde{\alpha}$ and $10^{12} \widetilde{k}^{2}$ for $\widetilde{\alpha}$ between 0 and 10 . The absolute maximum of the characteristic exponent for $n=1$ is $\mu_{k}=0.147$ at $\widetilde{\alpha}=0$ and $\widetilde{k}^{2}$ $=1.08 \times 10^{-13}$, in agreement with [6]. The maximum of the characteristic exponent for fixed $\widetilde{\alpha}$ decreases monotonically with $\widetilde{\alpha}$. In contrast, the width of the resonance band of the squared momentum $\widetilde{k}^{2}$ increases for $\widetilde{\alpha}$ small, reaching its maximum at $\widetilde{\alpha}=2.442$. In order to compare the efficiency of the particle production with the pure $\lambda \phi^{4} / 4$ model, we esti- 


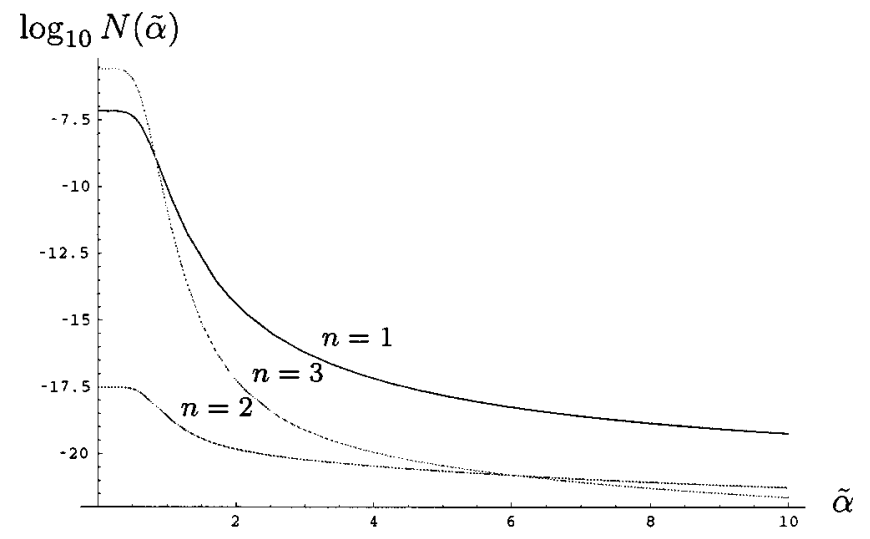

FIG. 5. Plot of $\log _{10} N(\widetilde{\alpha})$ as a function of $\widetilde{\alpha}$ for $n=1,2,3$.

mate the number density in units of $M_{P}^{3}$ after 30 oscillations of the inflaton field (when the back reaction becomes significant in the $\lambda \phi^{4}$ model) as

$$
N(\widetilde{\alpha}) \simeq \frac{1}{2 \pi^{2}} \int_{I} \widetilde{k}^{2} e^{120 \mu_{k} K(\kappa)} d \widetilde{k},
$$

where $I$ is the union of all the instability intervals. In Fig. 5 we plot the logarithm of the number density versus $\widetilde{\alpha}$. In Fig. 6 we represent the ratios $N(\widetilde{\alpha}) / N(0)$ for small values of $\widetilde{\alpha}$. The number density for $n=1$ (i.e., $g^{2}=\lambda$ ) is almost constant for $\widetilde{\alpha} \leq 0.2$. Therefore, in this case the effect of the term $\mu /\left(2 \phi^{2}\right)$ in the energy transfer from the inflaton field is negligible provided $\mu^{1 / 6} \lesssim 10^{-3} M_{P}$. The number density decreases sharply for $\widetilde{\alpha} \gtrsim 0.5$.

We next examine the case $n=2$. In this case the Lamé equation (9) possesses three instability zones, out of which the only relevant one is $4+m<\mathcal{E}<2(1+m$ $\left.+\sqrt{m^{2}-m+1}\right)$, leading to

$$
\frac{3 \lambda \widetilde{\alpha}^{2}(2-m)}{2 \pi D(m)}<\widetilde{k}^{2}<\frac{3 \lambda \widetilde{\alpha}^{2} \sqrt{m^{2}-m+1}}{\pi D(m)} .
$$

The width of the resonance band of the squared momentum $\widetilde{k}^{2}$ also increases in this case for small $\widetilde{\alpha}$, with its maximum

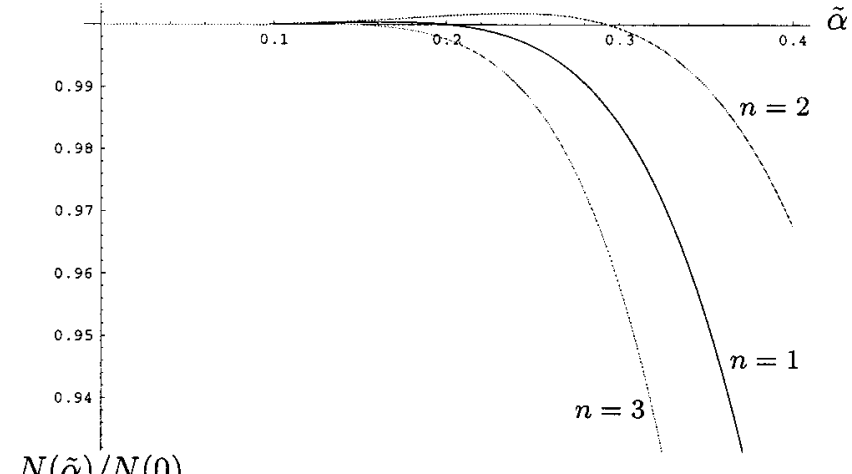

FIG. 6. Number densities ratio $N(\widetilde{\alpha}) / N(0)$ for small values of $\widetilde{\alpha}$ and $n=1,2,3$. located at $\widetilde{\alpha}=0.858$. The coefficients $a_{1}^{(2)}$ and $a_{2}^{(2)}$ defining the polynomial $M_{(2)}(z)$ are given by [omitting the superscript (2)]

$$
a_{1}=\frac{4-2 m-\mathcal{E}}{3 m}, \quad a_{2}=\frac{(1+m-\mathcal{E})(4+m-\mathcal{E})}{9 m^{2}} .
$$

One can immediately show that the roots of $M_{(2)}(z)$ are real and different if $\mathcal{E}$ lies in the above instability band. The coefficients $\beta_{1,2}$ and $D_{1,2}$ are respectively given by

$$
\begin{aligned}
& \beta_{1,2}=\frac{1}{2}\left(2+a_{1} \mp \sqrt{a_{1}^{2}-4 a_{2}}\right), \\
& D_{1,2}=\frac{ \pm\left(2+a_{1}\right)+\sqrt{a_{1}^{2}-4 a_{2}}}{2\left(1+a_{1}+a_{2}\right) \sqrt{a_{1}^{2}-4 a_{2}}},
\end{aligned}
$$

with $a_{1,2}$ given by Eq. (40). Note that $\beta_{1,2}^{-1}>1$ in the instability zone. The coefficient $C_{(2)}^{2}$ is given by

$$
\begin{aligned}
C_{(2)}^{2}= & \frac{1}{81 m^{4}}(1+m-\mathcal{E})(4+m-\mathcal{E})(1+4 m-\mathcal{E}) \\
& \times\left(\mathcal{E}^{2}-4(1+m) \mathcal{E}+12 m\right)
\end{aligned}
$$

The maximum of the characteristic exponent in the instability band also decreases monotonically with $\widetilde{\alpha}$. The absolute maximum is $\mu_{k}=0.036$ at $\tilde{\alpha}=0, \widetilde{k}^{2}=7.67 \times 10^{-13}$, four times smaller than the absolute maximum for $n=1$. Correspondingly, the particle production is also much less efficient than in the case $n=1$; see Fig. 5. The maximum particle production for $n=2$ occurs when $\widetilde{\alpha}=0.245$; see Fig. 6 .

The case $n=3$ presents some unexpected effects. In this case, the Lamé equation (9) possesses four instability zones, out of which the only relevant ones are $\mathcal{E}$ $\in\left(4(1+m), 2+5 m+2 \sqrt{4 m^{2}-m+1}\right) \quad$ and $\quad \mathcal{E} \in(5+2 m$ $\left.+2 \sqrt{m^{2}-m+4}, 5(m+1)+2 \sqrt{4 m^{2}-7 m+4}\right)$. In terms of the dimensionless momentum $\tilde{k}$, these are

$$
0<\widetilde{k}^{2}<\frac{3 \lambda \widetilde{\alpha}^{2}}{2 \pi D(m)}\left(m-2+2 \sqrt{4 m^{2}-m+1}\right),
$$

and

$$
\begin{aligned}
& \frac{3 \lambda \widetilde{\alpha}^{2}}{2 \pi D(m)}\left(1-2 m+2 \sqrt{m^{2}-m+4}\right) \\
& \quad<\widetilde{k}^{2}<\frac{3 \lambda \widetilde{\alpha}^{2}}{2 \pi D(m)}\left(1+m+2 \sqrt{4 m^{2}-7 m+4}\right) .
\end{aligned}
$$

For both resonance bands, the width increases for small $\widetilde{\alpha}$, with the respective maxima located at $\widetilde{\alpha}=1.052$ and $\widetilde{\alpha}$ $=0.533$. The coefficients of the polynomial $M_{(3)}(z)$ defined in Eq. (30) are given by

$$
a_{1}=\frac{9-6 m-\mathcal{E}}{5 m}
$$




$$
\begin{aligned}
a_{2}= & \frac{2 \mathcal{E}^{2}+(4 m-26) \mathcal{E}+27 m^{2}-51 m+72}{75 m^{2}}, \\
a_{3}= & -\frac{1}{225 m^{3}}\left(\mathcal{E}^{3}-2(4 m+7) \mathcal{E}^{2}\right. \\
& +[16 m(m+5)+49] \mathcal{E}-12(m+1)(8 m+3)) .
\end{aligned}
$$

The coefficients $C_{(3)}^{2}$ and $\beta_{i}, D_{i}, i=1,2,3$, are then easily obtained from Eqs. (32) and (33). The resulting expressions are very cumbersome and shall not be displayed here. It may be shown that the coefficients $\beta_{i}$ are all real and different in the resonance bands.

Just as in the previous cases $n=1,2$, the maximum of the characteristic exponent decreases monotonically with $\widetilde{\alpha}$ for both resonance bands. For the lower resonance band given in Eq. (43), the absolute maximum is $\mu_{k}=0.159$ at $\widetilde{\alpha}=0, \widetilde{k}^{2}$ $=2.09 \times 10^{-13}$, while for the higher one in Eq. (44) the maximum value is $\mu_{k}=0.0078$ at $\widetilde{\alpha}=0, \widetilde{k}^{2}=1.857 \times 10^{-12}$. The particle production at $\widetilde{\alpha}=0$ is two orders of magnitude more efficient than in the case $n=1$. However, it decreases with $\widetilde{\alpha}$ much faster than in the previous cases $n=1,2$; see Figs. 5 and 6.

\section{CONCLUSIONS}

In this paper we have characterized the most general scalar potential for the inflaton field leading to the Lamé equation for the matter field modes in a Minkowskian background. The resulting potential possesses a term of the form $\mu /\left(2 \phi^{2}\right)$ in addition to the terms $\lambda \phi^{4} / 4+K \phi^{2} / 2$ already studied in the literature. We have analyzed the effect of this new term in the preheating era after inflation in the particular case $K=0$. Exact expressions for the resonance bands and the characteristic exponents have been derived for certain values of the coupling constant between the inflaton and the matter fields. The effect of the new term in the particle production is virtually negligible provided $\mu^{1 / 6} \lesssim 10^{-3} M_{P}$, even though the inflaton potential is modified in an essential way near the origin. However, for $\mu^{1 / 6} \gg 10^{-3} M_{P}$ matter production is heavily suppressed by the new term as compared to the pure $\lambda \phi^{4} / 4$ model. The situation in this respect is expected to remain unchanged for other values of the coupling constant.

\section{ACKNOWLEDGMENTS}

A.L.M. wishes to thank J. García-Bellido for useful discussions. This work was partially supported by grants DGES PB98-0821 and DGICYT AEN97-1693.
[1] L. Kofman, A. Linde, and A. A. Starobinsky, Phys. Rev. Lett. 73, 3195 (1994).

[2] L. Kofman, A. Linde, and A. A. Starobinsky, Phys. Rev. D 56, 3258 (1997).

[3] Y. Shtanov, J. Traschen, and R. Brandenberger, Phys. Rev. D 51, 5438 (1995).

[4] J. H. Traschen and R. H. Brandenberger, Phys. Rev. D 42, 2491 (1990).

[5] P. B. Greene, L. Kofman, A. Linde, and A. A. Starobinsky, Phys. Rev. D 56, 6175 (1997).

[6] D. I. Kaiser, Phys. Rev. D 57, 702 (1998).

[7] D. Kaiser, Phys. Rev. D 59, 117901 (1999).

[8] W. H. Kinney and A. Riotto, Phys. Lett. B 435, 272 (1998).

[9] W. H. Kinney and A. Riotto, Astropart. Phys. 10, 387 (1999).

[10] E. D. Stewart, Phys. Lett. B 345, 414 (1995).
[11] J. D. Barrow and A. R. Liddle, Phys. Rev. D 47, R5219 (1993).

[12] I. Affleck, M. Dine, and N. Seiberg, Nucl. Phys. B256, 557 (1985).

[13] M. Abramowitz and I. Stegun, Handbook of Mathematical Functions (Dover, New York, 1965).

[14] W. Magnus and S. Winkler, Hill's Equation (Dover, New York, 1979).

[15] F. Finkel, A. González-López, and M. A. Rodríguez, J. Phys. A 33, 1519 (2000).

[16] If the $\chi$ field had a mass $m_{\chi}$, the only change in the above derivation would be to replace $k^{2}$ in Eqs. (8) and (12) by $k^{2}$ $+m_{\chi}^{2}$. These changes have no effect on Eq. (15).

[17] See also the forthcoming paper by J. García-Bellido for an extension of this method. 